\title{
EDITORIAL
}

A Revista Extensão \& Cidadania cumprindo o seu papel social junto à comunidade científica e público em geral, apresenta o número 14 , do volume 8 , composto por artigos científicos e relatos de experiência das ações extensionistas realizadas nas diferentes áreas de conhecimento, Ciências Exatas e da Terra; Ciências Biológicas, Engenharia/Tecnologia; Ciências da Saúde; Ciências Agrárias; Ciências Sociais; Ciências as Saúde; Ciências Agrárias; Ciências Sociais; Ciências Humanas e Linguística, Letras e Artes, presentes nas universidades públicas e privadas e nos institutos federais de ensino nas diferentes regiões do Brasil.

Esse número é composto por trabalhos produzidos em trinta instituições de Ensino Superior, localizadas em dez estados brasileiros: Bahia, Goiás, Minas Gerais, Paraná, Pernambuco, Rio de Janeiro, Rio Grande do Norte, Rio Grande do Sul, Santa Catarina e São Paulo. Os trabalhos estão organizados em duas partes, na primeira, são apresentados dezesseis textos científicos que versam temas relacionados ao campo do direito, da saúde, da educação e do ambiente; na segunda parte, Edição Especial sobre a Covid-19, os vinte e três textos científicos tratam especificamente das ações produzidas pelas universidades e institutos federais de ensino, visando ao cuidado com a saúde física, mental e emocional das pessoas no processo de distanciamento social, provocado pela pandemia.

Na primeira parte da edição, o artigo A "desjudicialização" do acesso à justiça como transformadora da realidade social da população: relatos de experiência do projeto de extensão Mediação e orientação sobre direitos na cidade de Feira de Santana

- BA, de Isa Helen Vieira de Jesus, desenvolvido através de uma ação extensionista realizada pela Universidade Estadual de Feira de Santana (UEFS), Bahia, apresenta os impactos do acesso à justiça, de forma extrajudicial, com a atuação do Escritório Popular de Mediação e Orientação sobre Direitos na gratuidade de documentos às pessoas hipossuficientes; consultoria judiciária e, também, com palestras e oficinas sobre temas jurídicos.

O artigo Reflexões sobre o Plantão Psicológico da Universidade Federal do 
Recôncavo da Bahia, de Edmar Henrique Dairell Davi, faz uma reflexão sobre as características do Plantão Psicológico e sua efetividade, a partir da análise dos prontuários dos pacientes atendidos no período de 2016 a 2018, na cidade de Santo Antônio de Jesus, na Bahia.

Em Revelar: a fotografia como ferramenta dialógica na concepção e no intercâmbio das narrativas visuais da cidade por meio de projetos de extensão, de Daniel P. Andrade, Henrique L. Araújo e Tamires P. Lima, realizado pela Universidade Federal Rural do Semi-Árido (UFERSA), Campus Pau dos Ferros, no Rio Grande do Norte, os autores fazem uma reflexão em torno das imagens urbanas, a partir dos paralelismos estabelecidos entre a fotografia e a cidade nos processos de representação e comunicação desses fenômenos.

$\mathrm{Na}$ sequência, os relatos de experiência reafirmam a concepção de extensão desse periódico ao tratarem de atividades relacionadas ao campo da cultura científica, técnica, ambiental, profissional e de gênero. O relato de experiência Aproveitamento de resíduos de tilápia como alternativa para o desenvolvimento local da comunidade do Açude Saco I, Serra Talhada-PE, de Juliana Maria Aderaldo Vidal-Campello, Ana Paula Gonçalves Silva, Danúbia Nunes dos Santos, Juliana Ferreira dos Santos e Weruska de Melo Costa, trata de uma ação realizada pela Universidade Federal Rural de Pernambuco (UFRPE), Unidade Acadêmica de Serra Talhada, Pernambuco, sobre o aproveitamento de escamas e pele de tilápia na confecção de peças artesanais apresentadas em portfólio, como uma fonte de renda complementando a atividade da pesca da região.

Em A produção textual no projeto de extensão "Filosofia no ensino fundamental: experiências filosóficas e a construção dos materiais didáticos" - uma experiência a muitas mãos, de Cleveron Montanarin, Karen Franklin da Silva e Raquel Aline Zanini, realizado pela Universidade Federal do Paraná (UFPR), apresenta o relato de experiência sobre o desenvolvimento de ações de construção dos materiais didáticos para o Ensino Fundamental I, no processo coletivo de produção textual fundamentado pelos textos clássicos da Filosofia, mediante discussões e atividades lúdicas.

O relato de experiência Elaboração e Aplicação de um recurso lúdico-didática visando à sensibilização ambiental relacionada à temática água, de Maria das Graças Cleophas e Raquel Rodrigues Dias, realizado pela Universidade Federal da Integração LatinoAmericana (UNILA), Foz do Iguaçu, Paraná, aborda sobre a aplicação de uma proposta

$$
\begin{array}{l|ll}
\multirow{2}{*}{\text { REVISTA }} & \multicolumn{2}{|l}{\text { EXTENSÃO \& CIDADANIA }} \\
\cline { 2 - 3 } & \text { v. 8, n. 14, p. 1-11, jul./dez. 2020. } & \text { ISSN 2319-0566 }
\end{array}
$$


lúdico-didática envolvendo a elaboração de um álbum de figurinhas Aquas tendo em vista o letramento científico e o estímulo à tomada de decisão frente às questões ambientais.

Em Formação e atuação sindical: como os processos de formação contribuem para o empoderamento das mulheres no meio rural, de Taise Pasa, Jane Francisca Richter da Costa e Zenicléia Angelita Deggerone, realizado pela Universidade Estadual do Rio Grande do Sul (UERGS), unidade de Erechim, e pelo Coletivo de Mulheres do Sindicato Unificado dos trabalhadores na Agricultura Familiar (SUTRAF-AU), que atua em 27 municípios da Região Alto Uruguai (RS), o relato de experiência enfatiza o protagonismo das mulheres de uma nova geração, na busca constante por empoderamento para reduzir as desigualdades de gênero existentes no meio rural.

O relato de experiência Humanizando a Medicina por meio da comunicação \& arte, de Edlaine Faria de Moura Villela e Cristian Junior da Costa, realizado pela Universidade Federal de Goiás (UFG) Regional Jataí, trata do fortalecimento da comunicação comunitária entre os alunos do curso de Medicina e a comunidade em um contexto cultural estimulando à socialização de conhecimento e às ações de alegria, compaixão e empatia, a fim de proporcionar uma formação humanizada e educação em saúde.

Em Obtenção de sabão para uso doméstico a partir de óleo usado de cozinha: uma experiência de extensão, de Thiago Muza Aversa, Everton Tomaz da Silva, Gabriela Fonseca de Mendonça, Queli Aparecida Rodrigues de Almeida e Suyane David Sá de Alvarenga, realizado pelo Instituto Federal de Educação, Ciência e Tecnologia do Rio de Janeiro (IFRJ Campus Duque de Caxias), trata de ações desenvolvidas para alunos, pais e comunidade do entorno com informações básicas acerca do descarte e reciclagem do óleo de cozinha.

O relato de experiência Oficinas temáticas sobre Química e cidadania: pesquisa, ensino e extensão, de Tathiane Milaré, Vitória Martoni Bueno Barbosa, Nara Flaviane Pistarini e Ana Carolina Dias de Oliveira, realizado pela Universidade Federal de São Carlos (UFSCar), campus de Araras, São Paulo, apresenta o desenvolvimento de oficinas que relacionam conhecimentos químicos aos aspectos sociais, políticos, econômicos e ambientais, com temas sobre química forense, cosméticos, alimentação e poluição ambiental.

O artigo $O$ papel da extensão na formação profissional, o caso da feira de trocas solidárias no IFRS, de Rodrigo Foresta Wolffenbüttel, realizado pelo Instituto Federal do Rio Grande do Sul (IFRS), analisa o papel de projetos de extensão na proposta de formação

\begin{tabular}{l|ll}
\multirow{2}{*}{ REVISTA } & EXTENSÃO \& CIDADANIA \\
\cline { 2 - 3 } & v. 8, n. 14, p. 1-11, jul./dez. 2020. & ISSN 2319-0566
\end{tabular}


profissional, investigando de que forma os projetos de extensão contribuem na promoção e no estabelecimento de novas dinâmicas de aprendizado, de saberes e de outras relações nos processos de educação profissional.

$\mathrm{O}$ artigo Percepções e comportamento financeiro de alunos do ensino médio do Vale do Taquari - RS, de Carla Rafaela Griebeler, Eloni José Salvi e Samuel Martim de Conto, realizado pelo Centro de Gestão Organizacional, da Universidade do Vale do Taquari (UNIVATES), de Lajeado, Rio Grande do Sul, aborda o comportamento e o conhecimento dos alunos do Ensino Médio da região do Vale do Taquari/RS em relação às finanças pessoais e a importância das escolas tratarem desse tema, visto que muitos alunos já possuem rendimentos e podem planejar o futuro sob essa perspectiva.

No relato de experiência Radiojornalismo educativo: experiências extensionistas na UESB FM, de Nayla Pereira dos Santos, realizado pela Universidade Estadual do Sudoeste da Bahia (UESB), campus de Vitória da Conquista, são abordadas as experiências extensionistas vividas pelos estudantes do curso de Comunicação Social com Habilitação em Jornalismo, na emissora UESB FM, uma rádio de cunho educativo.

O Relato de experiência: tecnologias sociais e práticas freireanas aplicadas na educação CTS, de Paula Simone Busko, realizado pela Universidade Federal de Santa Catarina (UFSC), Florianópolis, trata da inserção do tema Tecnologia Social no contexto dos estudos da Ciência, Tecnologia e Sociedade (CTS), relacionando práticas utilizadas em grupos de aprendizagem com os pressupostos do educador Paulo Freire como sugestão para novas proposições criativas. Propostas que possam atender às demandas sociais de determinadas localidades, como na educação, na cultura, na arte, no resgate ou no combate à vulnerabilidade do patrimônio cultural, imaterial ou histórico, na produção de energia limpa e na produção de alimentos e soluções para o uso de recursos naturais entre outros.

No relato de experiência Trabalhando a educação ambiental nas escolas públicas de Floresta - PE, Maria Aparecida de Sá Martins e Júlia Maria de Sá Carvalho, realizado pelo Instituto Federal do Sertão Pernambucano - PE (IF Sertão - PE), campus Floresta, Pernambuco, traz uma reflexão sobre a necessidade da escola educar ambientalmente seus alunos, mostrando a importância de saber utilizar os recursos naturais existentes de forma sustentável evitando o desperdício e o desrespeito à natureza.

Em "Vamos brincar?": relato de experiência do uso dos ambientes de aprendizagem na educação infantil, de Angélica Pereira de Oliveira, Fabíula Oliveira

$$
\begin{array}{l|ll}
\multirow{2}{*}{\text { REVISTA }} & \text { EXTENSÃO \& CIDADANIA } \\
\cline { 2 - 3 } & \text { v. 8, n. 14, p. 1-11, jul./dez. 2020. } & \text { ISSN 2319-0566 }
\end{array}
$$


Batista Mazeika e Sérgio Roberto Abrahão, realizado pela Universidade Federal do Paraná (UFPR), Curitiba, apresenta os resultados obtidos em dois Centros de Educação Infantil, localizados em Curitiba e na região metropolitana, com a construção do conhecimento relacionado à Linguagem Movimento, oportunizando subsídios teórico-práticos sobre a utilização de Ambientes de Aprendizagem, para a aplicabilidade nas creches, pré-escolas e escolas.

$\mathrm{Na}$ segunda parte deste número, na Edição Especial sobre a Covid-19, os artigos contemplam temáticas diversas que contribuem e contribuirão para os profissionais lidarem com a doença e com os problemas decorrentes. São ideias e modos de cuidar para prevenir e controlar a Covid-19, descritos por meio das experiências de profissionais de saúde, educadores, comunicadores, dentre outros, que podem ser replicados nos diversos cotidianos do Brasil, quiçá noutros países.

O texto Ação de extensão durante a pandemia da covid-19 impacta na formação discente - relato de experiência, de Crizane Hackbarth, Allan Guimarães Borçato e Eliziane Luiza Benedetti, do Instituto Federal de Santa Catarina (IFSC- São Carlos), Câmpus de São Carlos, apresenta os impactos na formação dos discentes extensionistas envolvidos na organização do 'I Webinário da Araucária e Erva-Mate', evento virtual que difundiu conhecimento científico sobre formas de manejo e técnicas de produção das espécies nativas Araucária (Araucaria angustifolia) e Erva-Mate (Ilex paraguariensis), através de palestras, atendendo à demanda de pessoas de diversos lugares, especialmente, do Rio Grande do Sul, Santa Catarina e Paraná, pelo fato de serem exploradas economicamente.

Em Ações do projeto Coral In'Canto da Universidade Federal de Uberlândia em tempos de pandemia, de Daniele do Espírito Santo Loredo da Silva, trabalho realizado pela Universidade Federal de Uberlândia (UFU), Campus Patos de Minas, Minas Gerais, trata da experiência de um coral não profissional que passou a desenvolver suas atividades de forma remota com encontros através da plataforma Hangout e Google Meet, a fim de levar a música aos diversos lugares com mensagens positivas nesse tempo de pandemia e isolamento social.

$\mathrm{Na}$ sequência, Ações extensionistas do IFSP São Roque no enfrentamento aos efeitos da Covid-19, de Gabriela Garcia Medeiros, Luiz Felipe Borges Martins, Mariana Neves Vieira, José Lucio da Silva Santos, João Daniel Matos e Christine Hauer Piekarz, do Instituto Federal de São Paulo (IFSP - São Roque), campus São Roque, apresenta as ações realizadas através lives e publicações em redes sociais abordando temas recorrentes, como:

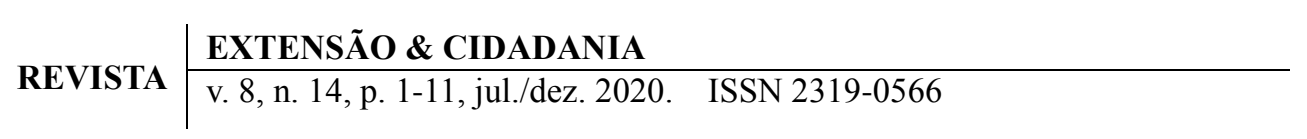


controle da ansiedade, prevenção ao suicídio, necessidade da manutenção das medidas preventivas da Covid-19 e a importância da prática de esportes durante o isolamento.

Em Ambientes virtuais de aprendizagem em contexto pandêmico: parceria entre universidades e escolas públicas da Bahia, de Marilete Calegari Cardoso, Socorro Aparecida Cabral Pereirae Antonete Araujo Silva Xavier, traz o relato de experiência do "Projeto de Extensão Ciber Ateliê Brincante: pesquisa formação sobre o brincar livre nos espaços escolares", em parceria com a Universidade Estadual do Sudoeste da Bahia (UESB), Universidade Federal da Bahia (UFBA) e Universidade do Estado da Bahia (UNEB), desenvolvido, neste período pandêmico, com a utilização do Ambiente Virtual de Aprendizagem (AVA), Moodle, e outras interfaces de interação e produção online.

Em A pandemia da Covid-19 na atenção primária à saúde: a extensão universitária atuando no enfrentamento, de Juliana Baldissera Dors, Tamíres Hillesheim Mittelmann, Eduarda Luiza Maciel da Silva, Victória Galletti dos Santos Arraes, Cesar Andres Diaz Arias e Graciela Soares Fonseca, da Universidade Federal da Fronteira Sul (UFFS), Campus Chapecó, Santa Catarina, apresenta as ações desenvolvidas pelo projeto de extensão "A pandemia do Coronavírus no território do Centro de Saúde da Família (CSF) Jardim América”, mediante duas frentes de atuação: Educação em Saúde direcionada para a comunidade e Educação Permanente em Saúde direcionada para os profissionais das equipes de saúde da família.

Em A utilização de podcasts para a conscientização sobre violência contra a pessoa idosa: relato de experiência, de Yasmin Sendrete de Carvalho Oliveira, Kamila Rangel Rosa, Mônica Cássia Bernardo de Souza, Daniela Braga Lima e Tábatta Renata Pereira de Brito, apresenta um trabalho realizado pelo Projeto de Extensão GerAções, da Universidade Federal de Alfenas (UNIFAL), Minas Gerais, que aborda a utilização de podcasts, hospedados na plataforma Anchor e divulgados pelas mídias sociais, como ferramenta para a difusão de informações a respeito da violência contra a pessoa idosa, sobretudo nesses tempos pandêmicos.

Cinema e infância: uma experiência extensionista em tempos de pandemia, de Luana de Gusmão Silveira e Marizete Bortolanza Spessatto, apresenta os resultados do projeto "IFSCineminha: arte e cultura na infância", desenvolvido pelo Instituto Federal de Educação de Santa Catarina (IFSC- Garopaba), no campus de Garopaba, que atende às escolas da rede pública de educação do município. O projeto evidencia que, ao difundir o 
cinema infantil, contribui-se diretamente para a educação integral e cidadã das crianças, mesmo em períodos em que as escolas estão de portas fechadas, como ocorreu neste ano de 2020, com a pandemia mundial do coronavírus.

No relato de experiência Consultoria ad hoc em saúde como estratégia de combate à pandemia pela Covid-19, de Thaís dos Santos de Souza, João Pedro Neves Pessoa, Sandra Rocha Gadelha Mello, Soraya Dantas Santiago dos Anjos e Vitória Solange Coelho Ferreira, realizado pela Universidade Estadual de Santa Cruz (UESC), Ilhéus, Bahia, apresenta os resultados obtidos da ação extensionista do Projeto "Consultoria ad hoc em saúde no combate à Covid-19”, bem como as inferências e vivências da equipe multidisciplinar, interdisciplinar e intersetorial responsável por sua execução.

Na sequência, Design de máscaras de segurança do tipo face shield: ações de enfrentamento à Covid-19 do IFSC de Itajaí, de Ulisses Filemon Leite Caetano, ação realizada pelo Instituto Federal de Santa Catarina (IFSC-Itajaí), campus de Itajaí, trata da produção de máscaras de proteção individual contra a Covid-19 que, após estudos, elegeu as máscaras do tipo face shield confeccionadas em PET Cristal por meio do processo de corte a laser para atender à demanda Centro Integrado de Gerenciamento de Riscos e Desastres da Regional de Itajaí (CIGERD) que oferta, gratuitamente, às pessoas que trabalham com atendimento ao público na região da Foz do Rio Itajaí como proteção facial a fim de diminuir as chances de contrair o coronavírus

No relato de experiência, Educação e promoção de cuidados no puerpério por meio de ferramentas online: um relato de experiência, de Raimundo Antonio Batista de Araújo, Artur Henrique Machado Lopes, Daniela Carvalho da Silva, Júlio César Ferreira, Suélem Maria de Sobral e Tárcio Augusto Medeiros de Alburquerque, realizado pela Universidade Federal de Campina Grande (UFCG), Paraíba, apresenta as práticas adotadas no projeto de extensão "Pandemia e puerpério: educação e promoção de cuidado", a partir boletins informativos dos programas de saúde presentes na comunidade, bem como no esclarecimento de dúvidas referentes ao período puerperal.

Em Ensino e extensão em Geociências versus Covid-19, de Luciano Alessandretti, Marco Antonio Delinardo da Silva e Felix Nannini, da Universidade Federal de Uberlândia (UFU), Minas Gerais, relata a experiência do projeto de extensão "Sedimentando conhecimentos geológicos na quarentena", que reinsere os discentes em suas atividades acadêmicas e populariza as Geociências por meio de lives com renomados pesquisadores e

$$
\begin{array}{l|ll}
\multirow{2}{*}{\text { REVISTA }} & \text { EXTENSÃO \& CIDADANIA } \\
\cline { 2 - 3 } & \text { v. 8, n. 14, p. 1-11, jul./dez. 2020. } & \text { ISSN 2319-0566 }
\end{array}
$$


profissionais liberais brasileiros e estrangeiros.

No relato de experiência Fale com obstetras - Campina Grande: relato de experiência de um projeto interprofissional, de Arthur Ferreira Cerqueira Amorim, Luanna Cristiny Domingos Nobre, Beatriz Berenguer de Souza Freitas, Maria Eduarda Gurgel Medeiros, Katarina Venâncio Antunes Romeu Ramos e Melania Maria Ramos de Amorim, da Universidade Federal de Campina Grande (UFCG), Paraíba, relata o Projeto "Fale com Obstetras", destacando as práticas realizadas por discentes de Medicina, através de dois aplicativos gratuitos para smartphones: o WhatsApp ${ }^{\circledR}$, para as orientações, e o Instagram ${ }^{\circledR}$, para os conteúdos informativos, ambos com enfoque na promoção à saúde de gestantes e puérperas.

Em Física e cultura científica moderna e contemporânea: um relato de experiência em meio à pandemia causada pelo coronavírus, de Alencar Migliavacca, Alison Vortmann dos Santos, Camila Gasparin e Willian Patrick Gonçalves, Instituto Federal de Educação de Santa Catarina (IFSC-Chapecó), campus de Chapecó, relata a experiência do projeto de extensão "Física e Cultura Científica Moderna e Contemporânea", no desenvolvimento das atividades como palestras online com a participação de convidados renomados, a fim de difundir conhecimentos relacionados à física para a comunidade externa e interna da instituição.

Na sequência, LAO-UNIFAL contra a Covid-19: um relato de experiência, de Larissa de Oliveira Briganti Carvalho, Alan Paulo Amaral Oliveira, Mariana Martins de Vitro, Renata Messias Frazão, Victor Alves Nascimento e Pollyanna Francielli de Oliveira, trata de uma ação desenvolvida pela Liga Acadêmica de Oncologia da Universidade Federal de Alfenas (LAO-UNIFAL), Minas Gerais, com a utilização das redes sociais como meio para a divulgação de informações científicas em linguagem popular sobre a Covid-19 e sua relação com a oncologia, de modo a combater notícias falsas por meio da disseminação de informações confiáveis.

Em O diálogo universidade-sociedade promovido por projetos de extensão em evolução, saúde e biodiversidade utilizando TDICs, de Luciana Aguilar-Aleixo e Gabriele Marisco, da Universidade Estadual do Sudoeste da Bahia (UESB), campus de Vitória da Conquista, relata a experiência das atividades extensionistas desenvolvidas a partir da parceria dos projetos "Popularizando a Ciência" e "Evolução para Todos", propostos como alternativas para a popularização do conhecimento científico e para a interlocução da

$$
\begin{array}{l|ll}
\multirow{2}{*}{\text { REVISTA }} & \multicolumn{2}{|l}{\text { EXTENSÃO \& CIDADANIA }} \\
\cline { 2 - 3 } & \text { v. 8, n. 14, p. 1-11, jul./dez. 2020. } & \text { ISSN 2319-0566 }
\end{array}
$$


comunidade acadêmica com a sociedade no contexto da pandemia da Covid-19, utilizando as Tecnologias Digitais de Informação e Comunicação (TDICs), como as mídias sociais e a rádio universitária, a fim de contribuir com a interlocução do conhecimento biológico, embasado no rigor científico.

Em O projeto de extensão "confecção de máscaras protetoras como medida de enfretamento da Covid-19", de Débora Pires Teixeira, Luanda dos Santos Alves, Consuelo Salvaterra Magalhães e Celina Angélica Lisboa Valente Carlos, da Universidade Federal Rural do Rio de Janeiro (UFRRJ), pela Unidade de Produção de Artigos Têxteis (UPAT), descreve o desenvolvimento de protótipos, que acompanham a normalização fornecida por órgãos competentes e todas as etapas de produção e distribuição das máscaras, bem como nas ações de capacitação online para os voluntários do projeto, elaborando métodos que se adaptem à situação pandêmica. Por meio do projeto, já foram confeccionadas e distribuídas mais de 10 mil máscaras aos funcionários e discentes da UFRRJ, Ong’s, ações solidárias, secretarias municipais de saúde e Instituições de Longa Permanência para Idosos, em diferentes municípios do estado do Rio de Janeiro.

No Projeto de extensão Vida Ativa - UNATI: relato de experiência durante a pandemia pela Covid-19, de Fernanda Cardoso Silva, Ítalo Gabriel Ferreira Germano, Nívea Akemi Ando Teixeira, Karla Caroline Oliveira, Rafaela de Oliveira Martins e Carolina Kosour, da Universidade Federal de Alfenas (UNIFAL), Minas Gerais, relata as ações desenvolvidas de forma remota para pessoas idosas durante o período de isolamento social ocasionado pela Covid-19. Os resultados apontaram repercussão positiva na manutenção funcional e melhoria da qualidade de vida dos idosos, além do impacto na formação do aluno promovendo os princípios da extensão: indissociabilidade, interdisciplinaridade, interação dialógica.

No Projeto Saberes no enfrentamento ao coronavírus - informação em tempo de pandemia - relato de experiência, de Greicy Cristine Kosvoski, Helena Fornari Basso e Margarete Dulce Bagatini, da Universidade Federal da Fronteira Sul (UFFS), Campus Chapecó, Santa Catarina, relata as ações do "Projeto saberes no enfrentamento ao coronavírus", executado por acadêmicos de Medicina e Enfermagem, através de informações diárias no Facebook, com a proposta de prevenir, orientar, atualizar e divulgar informações verídicas de cunho técnico-científico em relação ao novo coronavírus, proporcionando fácil entendimento ao público, tendo uma abrangência de repercussão em 8 estados brasileiros e

$$
\begin{array}{l|ll}
\multirow{2}{*}{\text { REVISTA }} & \multicolumn{2}{|l}{\text { EXTENSÃO \& CIDADANIA }} \\
\cline { 2 - 3 } & \text { v. 8, n. 14, p. 1-11, jul./dez. 2020. } & \text { ISSN 2319-0566 }
\end{array}
$$


em 12 países.

No relato de experiência Psicologia em link: projeto de escuta psicológica online em tempos de pandemia, de Odilza Lines de Almeida, Edisio Pereira da Silva Luz Júnior, Adriana Vespasiana Magalhães Dias, Joyce Fernandes Prates e Jeciana das Virgens Botelho, da Universidade Estadual do Sudoeste da Bahia (UESB), campus de Vitória da Conquista, apresenta a experiência da ação extensionista "Psicologia em Link", criada para desenvolver um espaço de escuta e acolhimento psicológico de forma online durante o período de afastamento social na pandemia da Covid-19 no ano de 2020.

Em Quarentena com afeto: um relato de experiência, de Natalia Tetemann Vilarim, Edmundo de Oliveira Gaudêncio, Artur Henrique Machado Lopes, Carla Cristina Garcia Barbosa, Daniela Carvalho da Silva e Thamiris Miranda Granja, da Universidade Federal de Campina Grande (UFCG), Paraíba, apresenta o relato de experiência acerca das ações sociais realizadas pelo Projeto "Quarentena com Afeto", disponibilizando acesso online e gratuito ao serviço de assistência à saúde mental para as pessoas que necessitem de ajuda e, também, a divulgação diária de informações confiáveis para o público geral, mantendo-se o foco nas pessoas consideradas vulneráveis e/ou em sofrimento psíquico por conta da atual epidemia.

Em A democratização do acesso à saúde em libras: um relato de experiência, de Karine Brito Matos Santos, Bruno Luz Pereira Santos, Maria Eduarda Silva Gomes Roberto, Maria Gabrielle Lopes Cordeiro, Rebeca Lima de Almeida Santos e Vinicius Gonçalves Rodrigues, da Universidade Estadual do Sudoeste da Bahia (UESB), campus de Vitória da Conquista, relata a experiência do evento "Saúde em Libras", realizado online e transmitido através da plataforma Youtube, sobre a importância da Língua de Sinais para uma comunicação eficaz e humanizada nos serviços de saúde, garantindo o acesso à saúde e o correto cumprimento dos princípios e diretrizes do SUS.

Em Utilização de vídeos como apoio social para residentes de instituição de longa permanência: relato de experiência, de Erika Cristina Napolitano Giuliano, Bárbara Assis da Silva, Laura Campos dos Santos e Flávia Della Lucia, da Universidade Federal de Alfenas (UNIFAL), Minas Gerais, trata-se de uma ação de extensão realizada pelo Projeto GerAções, componente da Universidade Aberta à Terceira Idade - UNATI em parceria com o Projeto NutrirArte, descreve a utilização de recursos de vídeo como forma de apoio social para os idosos residentes em uma Instituição de Longa Permanência para Idosos, durante o período de isolamento social causado pela pandemia da Covid-19, a fim de diminuir o impacto negativo

$$
\begin{array}{l|ll}
\multirow{2}{*}{\text { REVISTA }} & \multicolumn{2}{|l}{\text { EXTENSÃO \& CIDADANIA }} \\
\cline { 2 - 3 } & \text { v. 8, n. 14, p. 1-11, jul./dez. 2020. } & \text { ISSN 2319-0566 }
\end{array}
$$


da suspensão das visitas presenciais na instituição durante o período de isolamento social.

\section{Em Vírus: uma ameaça invisível - experiência de um curso EaD para} enfrentamento da Covid-19, de Renata de Souza Panarari, Vanessa Monteiro Eloi e Camila Clozato Lara, do Instituto Federal do Paraná (IFPR), Campus Paranavaí, trata-se de um relato de experiência acerca de um minicurso ofertado à comunidade interna e externa da instituição, com o objetivo de fornecer maiores esclarecimentos e conhecimento de base científica sobre as doenças virais, com enfoque ao novo coronavírus e à Covid-19, auxiliando, principalmente no combate aos fake news.

Assim, diante do negacionismo crescente ao conhecimento científico e dos ataques às universidades públicas brasileiras, a Revista Extensão \& Cidadania, nesse número em particular, com a Edição Especial sobre a Covid-19, reafirma a importância do desenvolvimento de projetos extensionistas que busquem a construção da coesão social e o aprofundamento da democracia; lutem contra a exclusão social e a degradação ambiental, bem como defendam a diversidade cultural e de gênero. Esperamos, portanto, que, no contexto da comunidade acadêmica, a leitura dos textos contribua para qualificar o diálogo sobre a necessidade de se garantir a indissociabilidade entre o ensino, a extensão e a pesquisa.

Vitória da Conquista, 18 de dezembro de 2020.

\section{Wotencure}

Marizete Argolo Teixeira

Professora Titular do Departamento de Saúde II, da UESB

Doutora em Enfermagem, pela UFBA

PhD. Université Aix-Marseille - França

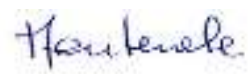

Luziêt Maria Fontenele Gomes

Professora Assistente do Departamento de Ciências Humanas e Letras, da UESB

Mestre em Letras: Educação e Cultura

Doutoranda em Memória: Linguagem e Sociedade, pela UESB

Editoras-chefes 